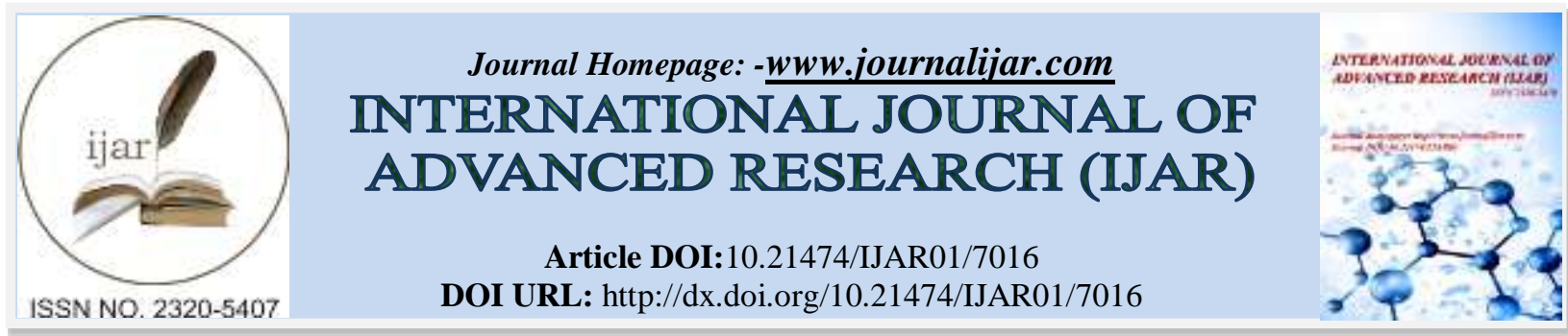

RESEARCH ARTICLE

\title{
SOLVE THE CAPACITANCE VEHICLE ROUTING PROBLEM ( CVRP) USING THE TABU SEARCH ALGORITHM (TS) AND THE PARTICLE SWARM OPTIMIZATION WITH PRACTICAL APPLICATION.
}

Dr. Abdul-Jabbar Khadr Bakhit ${ }^{1}$ and Ali ghani noori ${ }^{2}$.

1. Assistant Professor of Statistics at the University of Baghdad, College of Management and Economics.

2. Master Operations Research, University of Baghdad, College of Management and Economics.

\section{Manuscript Info}

Manuscript History

Received: 02 March 2018

Final Accepted: 04 April 2018

Published: May 2018

Keywords:-

Capacitated vehicle routing problem (CVRP), tabu search (TS) ,particle swarm optimization(PSO).

\begin{abstract}
In this paper we examine the vehicle routing problem (VRP) which is classified as a problem (NP-HARD). This problem addresses the design of tracks for a group of vehicles that equip a number of customers deployed in different locations. The objective of this problem is to reduce the total cost of delivery, taking into account the limitations of the problem, and also to design the optimal distribution of the tracks within the specified vehicle capacity limits. The study includes the most important methods used to solve the problem, the use of the tabu search algorithm (TS) and particle swarm optimization(pso) and the hybridization of a new algorithm that adopts the characteristics of tabu search with particle swarm optimization ( TS-PSO) in solving problem Issues. The performance and efficiency of the algorithms are compared by solving known issues of the problem. The results show that the tabu search algorithm (TS) is efficient in finding solutions to the problem. The ability to reach the mean deviation about (1.764) from the standard solutions for the set of standard issues. However, the TS-PSO is able to produce results that overcame the results of the tabu search algorithm as well as the particle swarm algorithm, although the hybrid algorithm requires double and large computational times compared to the two individually designed algorithms. The method of the problem has been applied in the General Company for the manufacture of grain distribution process from one of the mills to a group of agents deployed geographically The method was able to reach to draw and design the best possible paths for vehicles available at the least distance cut and the lowest number of vehicles.
\end{abstract}

Copy Right, IJAR, 2018,. All rights reserved.

\section{Introduction:-}

The field of transport and logistics services has become an important basis for the management and supply of goods and services in distribution systems and for a large number of real-world applications where institutions and commercial centers have begun to adopt procedures and systems that adopt planning in the management of logistics transport and Which will help in the effective changes in the reduction of the total costs. In fact, the transfer process represents a large proportion of the costs usually in addition to the cost of goods and services and the process of 
developing these methods helps to increase the integration of information systems in the production and commercial operations and logistics and the development of other factors and linking these Methods and tools that have been implemented in recent years that take into account most of the characteristics of distribution problems that have been applied in the real world.

And because of the breadth of economic fields, their need and necessity for consumers, which led to the emergence of trade exchange and the rapid spread of this trade of goods and transport requirements across regions and countries and the increase in the number of exported and imported goods and their transport requirements, the difficulty of planning and increasing the level of cost through strong competition between logistics providers makes it necessary to use methods based on On the mathematical methods and linear programming and with the help of the methods of software in planning the means of transport. From this concept arose the vehicle routing problem (VRP) and emerged many studies on this problem , These problems are categorized as NP-HARD. Which is related to the categories of transport problems, which aims to find the best tracks for a group of vehicles in order to achieve the demands of customers or service seekers. Where it was studied and research in the vehicle routing problems by many researchers in order to implement the formulas of this problem and to use them in solving the problems of the real world.

The problem concerns the existence of quantities required to be transported from a warehouse (a starting point for a group of vehicles with specific capacities for each vehicle) to the requesting parties and assigned to those quantities and the mechanism to guide the number of vehicles available in the existence of constraints generated and imposed the problem and choose and determine the best paths to achieve those quantities and chart those paths to achieve . Minimum distance for set tracks in stray conditions, The demand for each track does not exceed the capacity of each vehicle and that the demand for each node or customer is achieved through a single vehicle and that the number of tracks does not exceed the available vehicles. The goal is to reduce the overall cost of steering vehicles by steering the vehicle fleet consensually and optimizing the overall demand for vehicles by allocating the best and best route for each vehicle, reducing the number of vehicles used and minimizing distance by reducing dispersion of tracks.

\section{Reference review:-}

The idea of this problem was first raised by the scientists (G.Dantzing \& J. Ramser) in 1959 [6]. It then spread and became the focus of many researches and studies, where many studies and Researches related to this problem, especially in the years The latter was a major development in the methods of analyzing the problem and the methods of solution used in it. The research and studies that are specialized in methods and classification of the problem, and the introduction of a number of techniques that help in finding solutions to the Issues of the problem, followed a series of the most important studies that were put forward in recent years.

The study of( Shih-Wei Lin \& et al., 2009). To solve the vehicle routing problem through the hybridization algorithm based on the integration of the characteristics of Simulation Annealing algorithm and the tabu search algorithm where the performance of this algorithm was measured by testing on a set of standard issues and results found that the algorithm has reached solutions to the solutions of the standard known and efficient and comparable In previous studies using the same standard issues for (CVRP) [20].

The study of (Ben Peterson \& et al., 2010). They studied the routing of random-demand vehicles based on flexible operating mode. The main advantage of the proposed method is that it can exploit the correlation between demand fluctuations at the receiving sites and design a more robust uniform combination of charging methods. The study was applied to Bosch / In North America, the results and conclusions indicate that the cost reduced is up to $25 \%$ of the real costs, while the new method also achieves a comprehensive increase in efficiency in terms of demand fluctuations[23].

The study of (Lingling Du \& et al.,2012). They propose a new meta-heuristics algorithm to solving vehicle routing problem issues that combine the features of local search(LS) and Tabu search(TS). This algorithm is based on the generation of initial solutions through the first method. Solutions are improved and solutions are generated from the best solutions Through the second method, performance was tested through known standard issues of the problem and the method was applied practically to a real problem[7].

The study of (Juraj Fosin \& et al., 2014). Study the use of the Local Frequency Search (ILS) algorithm to be used to solve vehicle routing problem, Two methods were used to search for the algorithm. The first represents the 
binary substitution process within the same path. The second represents the binary substitution process between two different paths and the use of two types of strategies as a condition for stopping. The algorithm efficiency was proved by the computational experiments of a set of known issues, On the practical reality in the real world of a company in Ukraine[8].

5- The study of ( Shan Huen Huang ., 2015). Study and propose a method to solve the vehicle routing problem and determine the specific location of the multi-chamber capacity with the receiving and delivery routing with random request, This study is a mixture between the problem of positioning and the vehicle routing problem as the methodology of the solution has been divided into several stages starting in the stage of allocation of the site and then determine the contract or customers and then identify the best tracks linking the sites warehouses and customers, The tabu search algorithm (TS) was used to find solutions that identify and improve the best tracks and after applying the method and testing it through the use of standard issues that proved the approach of its ability and its possibilities in finding reasonable and good solutions[14].

6-the study of (Young Zhang \& et al.,2017). A study on the analysis of the problem of automatic vehicle routing in the field of logistics, taking into account the obstacles of the tracks, where they addressed the application of the use of vehicles in the field of logistics and the possibility of contributing to reduce the cost effectively through the logistics and reduce the potential risks in the reduction of tracks where the model was modified known to the vehicle routing problem and the use of the Particle Swarm algorithm after adding improvements on the basis of the proposed model. The technique was applied and prove its effectiveness and the possibility of applying the technique more broadly[27].

\section{Theoretical side:-}

\section{The concept of vehicle routing problem:-}

Know the vehicle routing problem (VRP) was the name given to a set of problems that include access and visit a contract (of customers) through a number of vehicles, which runs from the central repository and one or promised central warehouses[4].

This problem can be defined as the design of the least possible paths for a group of vehicles that start and return to a central warehouse which prepares a group of customers. Each customer is serviced by one vehicle. The total order for each track should not exceed the capacities and capacities of the vehicles. (VRP) is concerned with the distribution of goods and the service of the contract group (customers) through the fleet of vehicles, which starts from a center or a group of centers that stores the goods. These vehicles are routed through the adoption of a network of suitable roads. Planning and solving this problem calls for the identification of a set of roads to determine the road for each vehicle starts and ends through and check the vehicle allocated to each road all the applications allocated to that road and the lowest possible cost[22].

The capacitance vehicle routing problem (CVRP) is one of the most important problems of the problem. It is concerned with finding the best set of tracks for vehicles that reduce the distance of the total trip when a group of vehicles starts from a central warehouse that does not deliver a quantity of goods to a group of customers or points that request them. Goods and in specific vehicle capacities and then return these vehicles to the starting point where the framework for solving this problem is to comply with the following restrictions[25]:

Each customer is visited once by one vehicle only, Each vehicle departs and returns to the same point or center from which it started, The total demand of the customer group must not exceed the capacity specified for each vehicle in the track, The number of vehicles used in the distribution should not exceed the available number of vehicles.

The mathematical model for the capacity vehicle routing problem:-

$$
\begin{aligned}
& \text { Minimize } \sum_{i \in N} \sum_{\substack{j \in N \\
\text { Subject to: }}} \sum_{v \in V} C_{i j} X_{i j}^{v} \quad \ldots \ldots \ldots \\
& \sum_{v \in V} y_{i}^{v}=1 \quad \text { for } i \in N \quad \ldots \ldots \ldots
\end{aligned}
$$




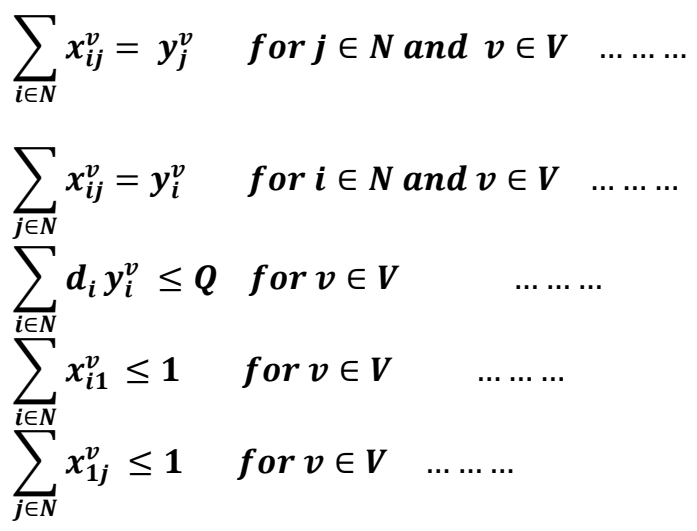

$\boldsymbol{x}_{\boldsymbol{i}}^{\boldsymbol{v}}$ Is a binary variable where it is $\left(\boldsymbol{x}_{\boldsymbol{i} j}^{\boldsymbol{v}}=1\right.$ ) If the node i Immediately preceded the node $\mathrm{j}$ on a vehicle trip v meaning that track from $\mathrm{i}$ to $\mathrm{j}$ Passed through and except this current $\left(\boldsymbol{x}_{\boldsymbol{i j}}^{v}=0\right), \boldsymbol{y}_{\boldsymbol{i}}^{v}$ Is a binary variable where it is $\left(y_{i}^{v}=1\right)$ if the node i served through the vehicle v Else then $\left(\boldsymbol{y}_{i}^{v}=0\right), \boldsymbol{C}_{i j}$ represent the distance(or cost) between the node I and the node $\mathrm{j}, \mathrm{N}$ representing all points (nodes) in addition to the warehouse $\mathrm{N}=(1,2, \ldots . ., \mathrm{n}), \mathrm{Q}$ represents the capacity of the vehicle $\mathrm{v}, \boldsymbol{d}_{\boldsymbol{i}}$ the demand for each node, $\mathbf{V}$ The number of available vehicles to provide service,

The equations above indicate that the equation(1) Is the function of the goal is to reduce the total distance cut or (reduce the total cost through the distance traveled), and that equation(2) Represents the constraint where each node or customer visits through only one vehicle, The equations (3)\&(4) It represents a constraint that every customer or node is a visit and leaves with the vehicle itself, the equation (5) the order that is assigned to the track must not exceed the specified vehicle capacity, The equations (6)\&(7) Constraints represent that the distribution satisfies the demands using available vehicles or less.

\section{Methods to resolve the problem:-}

There are three main types of solution methods that have been used to solve the problem since inception they are the exact methods and the Heuristics methods and Meta-heuristics .

\section{Exact methods:-}

The exact methods are among the first methods used to find solutions to the issues of vehicle routing problem (VRP). These methods include several types that have been used and different methods, most importantly (Branch and Bound)[26],( Cutting plane)[2],( Branch and Cut)[21], (Column generation)[3],( Branch \& Cut \& price)[9],( Dynamic programing)[17], In addition to other methods classified within the exact methods.

\section{Heuristics methods:-}

Initially used to solve the vehicle routing problem the exact methods but these methods have become often powerless to resolve this problem after the breadth of the problem and its constraints and increasing the breadth of the size of the problem and large areas of research into the solutions to the problem and thus it became difficult to obtain acceptable and reasonable solutions to issues, which are relatively large in some cases through the adoption of exact methods[18]. Therefore, there was a need to develop new methods that overcome the previous methods and here we developed the heuristics methods. heuristics methods have been introduced to solve optimization issues through many researchers(Clarke and J. Wright)[5], And increased interest in the development of their methods by other researchers, including (Gibert Laporte \& et al)[19]

\section{Meta-heuristics and Hybrid:-}

The meta-heuristics methods have proved effective after using them in solving compatibility problems and have found good results in most of these problems compared to the methods used previously. After the use of the solution of the problems of harmonized compatibility and used the exact methods and heuristics methods, which was able to help solve the issues of these problem, but the solutions that have been reached are not solutions desired, In 1986, the methods were developed by Glover, which was first called meta-heuristics and then the methods associated with this name were introduced to solve the compatibility problems, including the vehicle routing problem (VRP) 
Through the development of their types and their applications have been able to provide convincing solutions when implemented and semi-optimal in some cases during times of reasonable calculation [10].

\section{Tabu Search algorithm:-}

The first of the tabu search or banned research is the Glover in 1986. The tabu search is one of the meta-heuristic methods that are installed with other heuristics methods. The purpose of the method is to reduce the local optimization in the stages of the solution during Duplicates through strategy forbidden some moves[11]. This algorithm is a development of the local search algorithm LS) and was used to find good quality solutions for compatibility optimization problems. The main advantage of this algorithm is that it contains a constantly updated list that includes and stores the list of solutions that are reached in the solution stages. Each solution is given priority Or ambitious standard. The main components of the "Tabu list" are used to save the date and values for the development of the search process and this list allows to allow or reject the search in the neighborhoods that arise in the process of replication based on the information that is stored as a result of the recurrence and stored in the tabu list[24].

\section{A Tabu search algorithm Working mechanism:-}

The main inputs of tabu search are expressed as follows : the current solution, $\mathbf{S}^{*}$ Best known solution, $\mathbf{f}^{*}(\mathbf{s})$ The function value of the current best solution, $\mathbf{N}(\mathbf{s})$ Neighborhood of $\mathbf{S}, \mathbf{N}^{*}(\mathbf{s})$ the neighborhood or group accepted by the neighborhood $\mathbf{N}(\mathbf{s}), \mathbf{T}(\mathbf{L})$ Tabu list.

\section{Steps of Solution:-}

Step 1: Building the first solution S0

Step 2: We make $\mathrm{S}=\mathrm{S} 0, \mathrm{f} *=\mathrm{f}(\mathrm{s} 0), \mathrm{S} * \mathrm{~S} 0, \mathrm{TL}=Q$

Step 3: Search by data If the stop criterion is not met, the next step is taken;

Step 4: Solution $S$ is determined from a partial subset of the initial solution

Step 5: if $\mathrm{f}(\mathrm{s})<\mathrm{f}^{*}(\mathrm{~s})$ We make $\mathrm{f}^{*}(\mathrm{~s})=\mathrm{f}(\mathrm{s}), \mathrm{S}^{*}=\mathrm{S}$

Step 6: Update (TL) by adding specific moves or deleting the worst solutions when necessary[12].

\section{Particle Swarm Optimization algorithm:-}

Particle Swarm Optimization( PSO) is a community-based research tool that simulates the nature of individual movements in a swarm and uses them as a means of research. In the style of PSO, the best solutions are found for a particular problem using the behavior of a swarm of particles that Working as a search tool. The locations of the multi-dimensional particles are used to represent problem solutions. The Vector Velocities are used to represent the particle's search capability. Every step of repeating (PSO) consists of moving all the particles in the swarm from one site to another particle is evaluated has the possibility of solutions in various spaces of solutions to the problem. In the simulation of cognitive and social behavior of a swarm mechanism of action (PSO) is also working to save the information to the best sites for router for each particle. Where sites are known to give the function of the best target among the sites visited through the particle and there are the best global sites which is the best router among the routers of sites and thus the best and best global router is used to update the speed of particles[1].

The basic concepts of the algorithm particle swarm optimization (PSO)[15]:-

Cognitive Consistency: A pattern that connects particles in a given society and particles can represent any organisms with animal behavior that move groups according to a well thought out system. Cognitive consistency can be explained by studying the thinking of a group of particles to move in a particular way where the group thinks of particles in the same way or in a similar way.

\section{Position:-}

The site of the particle in different movements and frequencies.

Velocity:-

Are the vectors of the movement of the swarm particles of a specific society

Global Best: Is the optimal location that is obtained after an update is created through any particle of the Squadron

\section{Local Best:-}

The best solution that reaches the particle in the space of his movement 


\section{Neighborhood best:-}

Is the best solution to be found by the particle after experiencing the revival of neighboring solutions

\section{Swarm:-}

A group of community elements or so-called particle society-

\section{Social Influence:-}

A term used in the particle swarm optimization describes the behavior of particles where the idea is expressed as in real societies. Everyone has ideas and these ideas can change after experiments and social interactions as in conversations.

The same logic is used in the pso, where solutions are changed in the best way possible

\section{Swarm Intelligence:-}

Artificial intelligence that consists of simple factors that are composed and interact with one another and according to their environment.

* particle swarm optimization algorithm Framework (PSO) to solve the CVRP problem as in (Jin Ai \& et al ., 2009)[16].

\section{Practical side and numerical experiments:-}

In recent years, most studies and research have adopted the use of meta-heuristic methods in finding solutions to the vehicle routing problem because it was able to find acceptable solutions to the Issues of different sizes of the problem, noting that the problem the larger the size of the more complex and difficult to find standard solutions, In this context, the tabu heuristic algorithm (TS), which is an important meta-heuristic method, has been used to solve the CVRP problem, As well as the use of the pso algorithm and the hybridization of the two algorithms together and the formation of a hybrid algorithm (TS-PSO) and compare the performance through the use of a set of standard issues and then apply the algorithms to solve a real issue.

Implement algorithms and compare and analyze results through standard issues:-

In this aspect, the algorithms are implemented and their performance and efficiency are compared by solving a set of standard issues. Implementation is done through the use of the (Mat lab R2010b0 software and the use of a computer with (the Intel Celeron (R) CPU 1000M @ 1.80 GHz, RAM (2.00) GB) and computer system (Windows7 32 -bit), The set of standard issues available and known to CVRP is supported[13]. The selected issues consist of five groups $[\mathrm{A}, \mathrm{B}, \mathrm{E}, \mathrm{F}, \mathrm{P}]$ that differ in terms of vehicle capacity, number of vehicles, number of contract, As the contract for the issues used ranges between (16) to (135) knots and the number of vehicles ranges from (2) to (14) vehicles and vary the capacities of vehicles, both according to the units of the assumed quantity in each subset. The experience is promised occurrences when running the program for each issue were identified duplicates of the program between (10) to (15) to repeat the standard set of issues that are the performance of the program through which the test, It should be noted that the arithmetic time of the algorithms to link to the results to be presented to the set of standard issues in the frequency of the specified frequencies ranged from (1 - 72 hours) depending on the size of each issue. The computational results of the test shall be as in Tables (1)and (2) below ;

Table 1:-

\begin{tabular}{|c|c|c|c|c|c|c|c|c|c|c|c|c|}
\hline & \multirow{2}{*}{$\begin{array}{l}\text { Name } \\
\text { issue }\end{array}$} & \multicolumn{5}{|c|}{ TS } & \multicolumn{5}{|c|}{ PSO } & \multirow{2}{*}{$\begin{array}{c}\text { Standard } \\
\text { value }\end{array}$} \\
\hline & & GAP & MIN & MAX & $\begin{array}{c}\text { MEA } \\
\text { Obj }\end{array}$ & $\begin{array}{c}\text { N> } \\
\text { op } \\
\mathbf{t}\end{array}$ & GAP & MIN & MAX & $\begin{array}{c}\text { MEA } \\
\text { Obj }\end{array}$ & $\begin{array}{c}\mathbf{N}> \\
\text { op } \\
\mathbf{t}\end{array}$ & \\
\hline 1 & A-n32-k5 & 3.929 & 796 & 831 & 814.8 & $\mathbf{0}$ & 3.992 & 799 & 839 & 815.3 & 0 & 784 \\
\hline 2 & A-n33-k5 & 0.565 & 661 & 673 & 664.7 & 9 & 1.745 & 661 & 683 & 672.5 & 2 & 661 \\
\hline 3 & A-n45-k7 & 1.7740 & 1146 & 1207 & 1166 & 2 & 9.5870 & 1183 & 1291 & 1256 & $\mathbf{0}$ & 1146 \\
\hline 4 & A-n46-k7 & 3.0124 & 914 & 1001 & 942 & 1 & 11.1743 & 966 & 1043 & 1016 & $\mathbf{0}$ & 914 \\
\hline 5 & B-n31-k5 & 1.1409 & 672 & 692 & 680 & 4 & 2.0337 & 676 & 694 & 686 & $\mathbf{0}$ & 672 \\
\hline 6 & B-n35-k5 & 0.6771 & 955 & 981 & 961 & 9 & 1.7382 & 959 & 998 & 972 & 0 & 955 \\
\hline 7 & B-n45-k5 & 0.479 & 751 & 763 & 754.600 & 7 & 3.435 & 759 & 791 & 776.80 & $\mathbf{0}$ & 751 \\
\hline 8 & B-n45-k6 & 3.2842 & 681 & 733 & 700 & $\mathbf{0}$ & 9.8132 & 725 & 777 & 745 & $\mathbf{0}$ & 678 \\
\hline 9 & E-n22-k4 & 1.307 & 375 & 382 & 379.9 & 3 & $\begin{array}{l}0.373 \\
\end{array}$ & 375 & 382 & 376.4 & 8 & 375 \\
\hline 10 & E-n33-k4 & 0.7745 & 835 & 858 & 841.467 & 10 & 0.663 & 835 & 857 & 840.53 & 4 & 835 \\
\hline 11 & E-n51-k5 & 1.830 & 521 & 546 & 530.533 & 4 & 5.592 & 521 & 572 & 550.13 & 1 & 521 \\
\hline 12 & E-n76-k7 & 2.948 & 691 & 717 & 703.133 & $\mathbf{0}$ & 13.372 & 747 & 795 & 774.33 & $\mathbf{0}$ & 682 \\
\hline
\end{tabular}




\begin{tabular}{|c|c|c|c|c|c|c|c|c|c|c|c|c|}
\hline 13 & E-n76-k8 & 2.132 & 738 & 765 & 750.667 & $\mathbf{0}$ & 20.027 & 852 & 905 & 882.20 & $\mathbf{0}$ & 735 \\
\hline 14 & E-n101-k8 & 3.0192 & 821 & 868 & 841.667 & $\mathbf{0}$ & 24.268 & 985 & 1047 & 1015.27 & $\mathbf{0}$ & 815 \\
\hline 15 & E-n101-k14 & 4.413 & 1092 & 1157 & 1124.533 & $\mathbf{0}$ & 42.643 & 1471 & 1584 & 1536.27 & $\mathbf{0}$ & 1067 \\
\hline 16 & F-n45-k4 & 0.5064 & 724 & 730 & 728 & 5 & 1.1050 & 724 & 742 & 732 & 4 & 724 \\
\hline 17 & F-n72-k4 & 1.294 & 237 & 244 & 240.067 & 5 & 6.160 & 244 & 259 & 251.60 & $\mathbf{0}$ & 237 \\
\hline 18 & F-n135-k7 & 8.055 & 1194 & 1296 & 1255.6 & $\mathbf{0}$ & 43.115 & 1613 & 1703 & 1663 & $\mathbf{0}$ & 1162 \\
\hline 19 & P-n16-k8 & 0.163 & 450 & 461 & 450.7 & 14 & $\mathbf{0}$ & 450 & 450 & 450 & 15 & 450 \\
\hline 20 & P-n19-k2 & $\mathbf{0}$ & 212 & 212 & 212 & 15 & $\mathbf{0}$ & 212 & 212 & 212 & 15 & 212 \\
\hline 21 & P-n20-k2 & 0 & 216 & 216 & 216 & 15 & $\mathbf{0}$ & 216 & 216 & 216 & 15 & 216 \\
\hline 22 & P-n21-k2 & $\mathbf{0}$ & 211 & 211 & 211 & 10 & 0 & 211 & 211 & 211 & 10 & 211 \\
\hline 23 & P-n22-k2 & $\mathbf{0}$ & 216 & 216 & 216 & 15 & $\mathbf{0}$ & 216 & 216 & 216 & 15 & 216 \\
\hline 24 & P-n23-k8 & 0.2268 & 529 & 537 & $\mathbf{5 3 0}$ & 12 & 1.2350 & 529 & 541 & 536 & 2 & 529 \\
\hline 25 & P-n40-k5 & 1.3100 & 458 & 479 & 464 & 6 & 4.5560 & 470 & 492 & 479 & $\mathbf{0}$ & 458 \\
\hline 26 & P-n45-k5 & 1.190 & 510 & 527 & 516.067 & 4 & 3.229 & 516 & 539 & 526.47 & $\mathbf{0}$ & 510 \\
\hline 27 & P-n50-k7 & 2.900 & 560 & 584 & 570.067 & $\mathbf{0}$ & 9.711 & 586 & 633 & 607.80 & $\mathbf{0}$ & 554 \\
\hline 28 & P-n50-k8 & 2.45113 & 631 & 657 & 646.466 & 1 & 11.928 & 687 & 738 & 706.2 & 0 & 631 \\
\hline \multicolumn{2}{|c|}{ General average } & 1.764 & $\begin{array}{c}635.6 \\
071\end{array}$ & & 646.8203 & & 8.268 & $\begin{array}{c}685.28 \\
57 \\
\end{array}$ & & 704.35 & & 632.1786 \\
\hline
\end{tabular}

Table 2:-

The measures shown in Tables 1 and 2 Indicate the results obtained when implementing and running the algorithms to solve standard Issues. The performance analysis of the algorithms is performed through the following measurements ; (Min obj) The lowest value is obtained during the specific frequencies, (Max obj) The highest value obtained during the specified frequencies, (Mea obj) The average values of solutions for the specified

\begin{tabular}{|c|c|c|c|c|c|c|c|}
\hline & \multirow{2}{*}{ Name issue } & \multicolumn{5}{|c|}{ PSO-TS } & \multirow{2}{*}{ Standard value } \\
\hline & & GAP & MIN & MAX & $\begin{array}{c}\text { MEA } \\
\text { Obj }\end{array}$ & N>opt & \\
\hline 1 & A-n32-k5 & 2.474 & 796 & 827 & 803.4 & 0 & 784 \\
\hline 2 & A-n33-k5 & 0 & 661 & 661 & 661 & 15 & 661 \\
\hline 3 & A-n45-k7 & 0.5880 & 1146 & 1170 & 1153 & 2 & 1146 \\
\hline 4 & A-n46-k7 & 0.2334 & 914 & 928 & 916 & 11 & 914 \\
\hline 5 & B-n31-k5 & 0.0893 & 672 & 681 & 673 & 14 & 672 \\
\hline 6 & B-n35-k5 & 0.0000 & 955 & 955 & 955 & 15 & 955 \\
\hline 7 & B-n45-k5 & 0.044 & 751 & 754 & 751.33 & 13 & 751 \\
\hline 8 & B-n45-k6 & 1.1406 & 678 & 708 & 686 & 6 & 678 \\
\hline 9 & E-n22-k4 & 0 & 375 & 375 & 375 & 10 & 375 \\
\hline 10 & E-n33-k4 & 0.032 & 835 & 839 & 835.27 & 14 & 835 \\
\hline 11 & E-n51-k5 & 0.473 & 521 & 531 & $\mathbf{5 2 3 . 4 7}$ & 9 & 521 \\
\hline 12 & E-n76-k7 & 1.884 & 690 & 704 & 695.87 & $\mathbf{0}$ & 682 \\
\hline 13 & E-n76-k8 & 0.916 & 737 & 749 & 741.73 & $\mathbf{0}$ & 735 \\
\hline 14 & E-n101-k8 & 1.705 & 820 & 843 & 830.93 & $\mathbf{0}$ & 815 \\
\hline 15 & E-n101-k14 & 2.953 & 1100 & 1132 & 1108.80 & $\mathbf{0}$ & 1067 \\
\hline 16 & F-n45-k4 & 0.0921 & 724 & 730 & 725 & 13 & 724 \\
\hline 17 & F-n72-k4 & 0.253 & 237 & 241 & 237.60 & 12 & 237 \\
\hline 18 & F-n135-k7 & 3.262 & 1180 & 1228 & 1199.90 & 0 & 1162 \\
\hline 19 & P-n16-k8 & $\mathbf{0}$ & 450 & 450 & 450 & 15 & 450 \\
\hline 20 & P-n19-k2 & 0 & 212 & 212 & 212 & 15 & 212 \\
\hline 21 & P-n20-k2 & $\mathbf{0}$ & 216 & 216 & 216 & 15 & 216 \\
\hline 22 & P-n21-k2 & 0 & 211 & 211 & 211 & 10 & 211 \\
\hline 23 & P-n22-k2 & 0 & 216 & 216 & 216 & 15 & 216 \\
\hline 24 & P-n23-k8 & 0 & 529 & 529 & 529 & 15 & 529 \\
\hline 25 & P-n40-k5 & 0.1892 & 458 & 467 & 459 & 12 & 458 \\
\hline 26 & P-n45-k5 & 0.183 & 510 & 515 & 510.93 & 11 & 510 \\
\hline 27 & P-n50-k7 & 0.818 & 554 & 565 & 558.53 & 1 & 554 \\
\hline 28 & P-n50-k8 & 0.70787 & 631 & 648 & 635.4 & 6 & 631 \\
\hline & eral average & 0.644 & 634.9643 & & 638.22 & & 632.1786 \\
\hline
\end{tabular}

frequencies are calculated by the following equation

$$
\text { Mea } \text { obj }=\frac{\text { Frequency value } 1+\text { Frequency value } 2+\cdots+\text { Frequency value } n}{n} \ldots
$$


(N - opt) The number of times to get the standard solution during the specified frequencies, (opt) The standard optimum value is known for the issue, (Gap) It is a measure of the performance ratio between the average values of solutions and the known standard value of the issue and is produced by the following equation

Gap $=\frac{\text { mea obj }- \text { opt }}{\text { lopt }} \times 100 \% \quad \ldots \ldots \ldots$

In the results obtained for the set of standard issues we notice that the solution values for the issues vary from one question to another depending on the size of the issue, If the question (p-n16-k8) is characterized by the number of small nodes and the number of vehicles compared to the average range of issues where the results from Table (1) show that the tabu search algorithm (TS) reached standard solutions (14) and with a relatively simple gap from the average solution. For the same issue, the other algorithms were able to reach $(15,15)$ times the standard solutions for the( PSO, TS-PSO) algorithms, respectively, as shown in Tables (1) and (2) during (15) program repetitions.

And for the issue (B-n45-k5), it is characterized by the number of medium nodes relative to the set of issues and the number of vehicles averaged. During 15 repetitions, the TS algorithm could reach (7) times the standard solution and the average gap of the values of the frequencies of the standard value of the problem It was (Mea obj $=0.479)$ where it was able to reach (751) the best value which represents (min obj) during the specific repetition process and get value (763) as the highest value which represents (max obj), If we look at issues of greater magnitude in the issue (E-n101-k8) the tabu search algorithm (TS) can reach the average difference of values from the standard value of the issue by (3.019). In general, we notice that the relative gap of the average solutions for each issue increases as the issue grows, from the total computational result of the set of questions shows that the (TS) algorithm has been able to obtain results that overcome the particle swarm algorithm (pso) during the experiments in Table (1), The hybrid algorithm ( TS-PSO), whose results are shown in Table(2), has relatively outperformed the (TS) algorithm in some tested issues. Where the average general deviation of the set of issues tested was $(8.268,1.764,0.644)$ for the algorithms (PSO, TS, TS-PSO), respectively . In addition, the TS algorithm has an arithmetic time of operation that is relatively acceptable and reasonable compared with the TS-PSO, which requires large computational times to find solutions.

\section{The practical application of the vehicle routing problem in the real world:-}

The proposed method of solving CVRP problem is applied to solve a real problem applied by the researcher where the proposed algorithms will be applied to solve the problem after testing and proving its efficiency in finding solutions to the problem which are designed according to the model and constraints of the problem studied. Apply the supported algorithms to find the best tracks for vehicles and achieve the least possible distances for the distribution of vehicles in the best possible paths, Where the study of distributions of the General Company for the manufacture of grain in Iraq, one of the formations of the Ministry of Commerce and the identification of a sample during the selection of mill Supply a number of agents deployed geographically in Baghdad.

Information is built for the array of spaces between agents using the use of the Hacks feature (Google).

And through the basic information obtained and prepared by the researcher, which includes the name of the agent and geographical location and the quantities specified for each agent as indicated by the carrier and can be described as follows: The number of agents is (69) agent and is related to processing the request to them through one mill and The number of vehicles available at the company is 10 homogeneous vehicles with the same capacity, the capacity of each vehicle is $(35000 \mathrm{~kg})$ and the total demand for all agents is $(253451 \mathrm{~kg})$ and the quantities distributed to the agents from 1 to 69 agent.

\section{The Solution and analysis of results:-}

The algorithms used for the solution are implemented after they have been used to solve experimental issues to solve the practical problem of applying the CVRP problem in the General Grain Manufacturing Company. After the implementation of the program for algorithms, the results appear in the following tables (3) and (4)

Table 3:-

\begin{tabular}{|c|c|c|c|c|c|}
\hline \multicolumn{2}{|c|}{ Particle Swarm algorithm running } & & \multicolumn{3}{c|}{ Tabu search algorithm running } \\
\hline $\begin{array}{c}\text { Amount } \\
\text { assigned } \\
\text { to the }\end{array}$ & Elements path & path & $\begin{array}{c}\text { Amount } \\
\text { assigned } \\
\text { to the }\end{array}$ & Elements path & path \\
\hline
\end{tabular}




\begin{tabular}{|c|c|c|c|c|c|}
\hline route & & & route & & \\
\hline 34424 & $\begin{aligned} 1 \rightarrow 31 & \rightarrow 30 \rightarrow 45 \rightarrow 56 \rightarrow 24 \\
& \rightarrow 26 \rightarrow 41 \rightarrow 1\end{aligned}$ & R1 & 31758 & $\begin{aligned} 1 \rightarrow 53 & \rightarrow 19 \rightarrow 54 \rightarrow 18 \rightarrow 16 \rightarrow 14 \rightarrow \\
57 \rightarrow 67 & \rightarrow 64 \rightarrow 66 \rightarrow 65 \rightarrow 2 \rightarrow 1\end{aligned}$ & R1 \\
\hline 29511 & 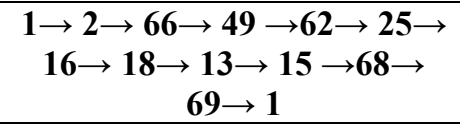 & $\mathbf{R 2}$ & 34759 & $\begin{aligned} 1 \rightarrow 52 \rightarrow 63 & \rightarrow 49 \rightarrow 68 \rightarrow 55 \rightarrow 59 \\
& \rightarrow 62 \rightarrow 1\end{aligned}$ & $\mathrm{R} 2$ \\
\hline 26323 & $1 \rightarrow 70 \rightarrow 5 \rightarrow 52 \rightarrow 1$ & R3 & 34299 & $1 \rightarrow 20 \rightarrow 21 \rightarrow 5 \rightarrow 3 \rightarrow 44 \rightarrow 60 \rightarrow 1$ & R3 \\
\hline 34575 & $1 \rightarrow 11 \rightarrow 48 \rightarrow 44 \rightarrow 1$ & R4 & 34811 & $1 \rightarrow 48 \rightarrow 7 \rightarrow 6 \rightarrow 12 \rightarrow 8 \rightarrow 11 \rightarrow 1$ & $\mathrm{R} 4$ \\
\hline 34787 & $\begin{aligned} & 1 \rightarrow 60 \rightarrow 55 \rightarrow 64 \rightarrow 65 \rightarrow 67 \rightarrow \\
& 10 \rightarrow 59 \rightarrow 1\end{aligned}$ & $\mathbf{R 5}$ & 34588 & $\begin{array}{c}1 \rightarrow 47 \rightarrow 51 \rightarrow 50 \rightarrow 58 \rightarrow 13 \rightarrow 10 \rightarrow \\
15 \rightarrow 4 \rightarrow 17 \rightarrow 43 \rightarrow 1\end{array}$ & R5 \\
\hline 34852 & $\begin{aligned} & 1 \rightarrow 43 \rightarrow 47 \rightarrow 63 \rightarrow 50 \rightarrow 53 \rightarrow \\
& 6 \rightarrow 20 \rightarrow 21 \rightarrow 3 \rightarrow 8 \rightarrow 7 \rightarrow 12 \\
& \quad \rightarrow 61 \rightarrow 42 \rightarrow 37 \rightarrow 1\end{aligned}$ & R6 & 34700 & 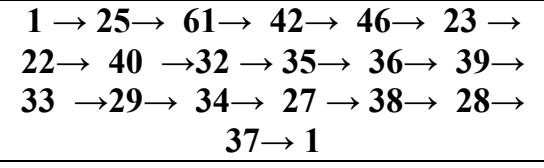 & R6 \\
\hline 31738 & $\begin{aligned} 1 \rightarrow 51 & \rightarrow 58 \rightarrow 14 \\
19 & \rightarrow 57 \rightarrow 54 \rightarrow 17 \rightarrow 9 \rightarrow 1\end{aligned}$ & R7 & 29227 & $\begin{aligned} 1 \rightarrow 69 \rightarrow 70 & \rightarrow 26 \rightarrow 41 \rightarrow 24 \rightarrow 56 \\
\rightarrow 1 & \rightarrow 4\end{aligned}$ & R7 \\
\hline 27241 & $\begin{array}{c}1 \rightarrow 46 \rightarrow 23 \rightarrow 22 \rightarrow 32 \rightarrow 40 \\
\rightarrow 34 \rightarrow 33 \rightarrow 29 \rightarrow 35 \rightarrow 36 \rightarrow \\
39 \rightarrow 27 \rightarrow 38 \rightarrow 28 \rightarrow 1\end{array}$ & R8 & 19309 & $1 \rightarrow 9 \rightarrow 45 \rightarrow 30 \rightarrow 31 \rightarrow 1$ & R8 \\
\hline & obj function= 469 & & & obj function $=442$ & \\
\hline
\end{tabular}

Table (4):-

\begin{tabular}{|c|c|c|}
\hline \multicolumn{3}{|c|}{ Pso - Tabu search algorithm running } \\
\hline $\begin{array}{c}\text { Amount } \\
\text { assigned to } \\
\text { the route }\end{array}$ & Elements path & path \\
\hline 23687 & $1 \rightarrow 28 \rightarrow 38 \rightarrow 27 \rightarrow 34 \rightarrow 29 \rightarrow 33 \rightarrow 39 \rightarrow 36 \rightarrow 35 \rightarrow 32 \rightarrow 40 \rightarrow 22 \rightarrow 25 \rightarrow 1$ & R1 \\
\hline 25796 & $1 \rightarrow 5 \rightarrow 3 \rightarrow 44 \rightarrow 1$ & R2 \\
\hline 34965 & $1 \rightarrow 11 \rightarrow 7 \rightarrow 6 \rightarrow 58 \rightarrow 9 \rightarrow 1$ & R3 \\
\hline 31291 & $1 \rightarrow 43 \rightarrow 51 \rightarrow 45 \rightarrow 24 \rightarrow 42 \rightarrow 61 \rightarrow 41 \rightarrow 23 \rightarrow 46 \rightarrow 37 \rightarrow 1$ & R4 \\
\hline 31528 & $1 \rightarrow 56 \rightarrow 50 \rightarrow 63 \rightarrow 49 \rightarrow 47 \rightarrow 62 \rightarrow 69 \rightarrow 70 \rightarrow 1$ & R5 \\
\hline 30172 & $1 \rightarrow 52 \rightarrow 59 \rightarrow 18 \rightarrow 16 \rightarrow 14 \rightarrow 54 \rightarrow 19 \rightarrow 4 \rightarrow 53 \rightarrow 17 \rightarrow 8 \rightarrow 1$ & R6 \\
\hline 27797 & $1 \rightarrow 20 \rightarrow 21 \rightarrow 12 \rightarrow 68 \rightarrow 55 \rightarrow 26 \rightarrow 30 \rightarrow 31 \rightarrow 1$ & R7 \\
\hline 28576 & $1 \rightarrow 60 \rightarrow 48 \rightarrow 66 \rightarrow 65 \rightarrow 2 \rightarrow 1$ & R8 \\
\hline 19639 & $1 \rightarrow 10 \rightarrow 15 \rightarrow 13 \rightarrow 67 \rightarrow 64 \rightarrow 57 \rightarrow 1$ & R9 \\
\hline & obj function $=438$ & \\
\hline
\end{tabular}

The results shown in Table (3) indicate that the forbidden search algorithm (TS) was reached by solving the value (442), which is the least distance achievable during the specific frequencies. The result is the construction of (8) paths of each path starting and ending in mill Each path is a road for one vehicle. The quantities for each track indicate that the vehicle number (1) has passed through 12 agents and reached the quantity determined for them $(31758 \mathrm{~kg}$ ) and that the vehicle No. (2) has passed by (7) agents and achieved the amount assessed for them (34759 $\mathrm{kg}$ ) and the vehicle No.(3) has covered (6) agents and reached the amount determined by them $(34299 \mathrm{~kg})$ and the vehicle No. (4) has passed (6) and achieved the specific request them (34811 kg) and cart No. (5) passed by (10) agents and achieved the amount assessed for them $(34588 \mathrm{~kg})$ as well as vehicles $(6,7$ and 8$)$. Each of these vehicles passed by (18, 6 and 4) agents respectively, and their specific orders were met with $(34700,29227$ and $19309 \mathrm{~kg})$ respectively.

We note that the algorithms used in the solution were able to find solutions to the problem. The (PSO) algorithm for the product (469) allocated 8 paths as in Table (3) and the value of the TS-PSO hybrid algorithm is (438) through (9) paths as we observe in Table (4). The results indicate that the TS algorithm has been able to obtain a better value than the(PSO) algorithm. As in numerical experiments, the( TS-PSO )has overcome the tabu search algorithm (TS) as it has been able to achieve a relatively better value Of the value obtained using the tabu Search algorithm (TS). 


\section{Conclusions:-}

1. Numerical experiments show that the TS algorithm is efficient and efficient in solving CVRP problems. The mean relative gap between algorithm values and standard solutions was about (1.764) for the set of standard problem issues.

2. The CVRP problem is becoming more complex as the problem becomes larger and the problem becomes more difficult to find. This is evidenced by the observation of solutions to standard issues where we note that the larger the size of the gap the greater the gap of the standard solution known.

3. The use of hybridization of the tabu search algorithm with the particle swarm algorithm is better in finding solutions to the Issues of the problem, but the necessary arithmetic time is relatively large compared to the use of the single-tabu search algorithm to solve problem Issues.

\section{Recommendations:-}

1. In this research, the CVRP method is used for a single depot(mill) and a set of specific agents. The method can be generalized to include the milling group at the General Grain Processing Company and a large number of agents associated with the company and the number of vehicles available to the company, With the General Company for the transfer of flour and other products in the company. Thus adoption of the method and generalization can generate a stable and clear strategy can be adopted monthly in the distribution process best distances and paths are allocated and the lowest possible cost.

2. VRP can be applied in other strategic distribution areas to distribute applications to public and private sector companies such as pharmaceuticals, agricultural crops, garbage collection, street cleaning and other logistics related to vehicle routing. Trying to expand the problem and its restrictions to include additional restrictions on vehicle capacity restrictions such as windows The time and distance specified for each vehicle, the probability of meeting potential demands, the fog, the heterogeneity of the fleet of vehicles, the achievement of demands that exceed the capacity of vehicles, and other variables and constraints that arise in the vehicle routing problem.

3. It is recommended to try to find a method used to solve the problem is not affected by the size and type of issues for the problem of steering the vehicle (VRP) although the algorithm used to solve the problem was relatively small gap for the results of the standard solutions, but the results obtained are affected by the size of the issue where the larger The size of the issue increased the gap between the standard solution and the results of the algorithm.

\section{References:-}

1. Ai, J. and V. Kachitvichyanukul. A study on adaptive particle swarm optimization for solving vehicle routing problems. in The 9th Asia Pacific industrial engineering and management systems conference. 2008

2. Belenguer, J.M. and E. Benavent, A cutting plane algorithm for the capacitated arc routing problem. Computers \& Operations Research, 2003. 30(5): p. 705-728

3. Choi, E. and D.-W. Tcha, A column generation approach to the heterogeneous fleet vehicle routing problem.Computers \& Operations Research, 2007. 34(7): p 2080-2095

4. Christofides, N., The vehicle routing problem. Revue française d'automatique, informatique, recherche opérationnelle. Recherche opérationnelle, 1976. 10(V1): p. 55-70.

5. Clarke, G. and J.W. Wright, Scheduling of vehicles from a central depot to a number of delivery points. Operations research, 1964. 12(4): p. 568-581

6. Dantzig, G.B. and J.H. Ramser, The truck dispatching problem.Management science, $\quad$ 1959. 6(1): p. 80-91

7. Du, L. and R. He, Combining nearest neighbor search with tabu search for large- scale vehicle routing problem. Physics Procedia, 2012. 25: p. 1536-1546

8. Fosin, J., T. Carić, and E. Ivanjko, Vehicle Routing Optimization Using Multiple Local Search Improvements. automatika, 2014. 55(2): p. 124-132

9. Fukasawa, R., et al., Robust branch-and-cut-and-price for the capacitated vehicle routing problem. Mathematical programming, 2006. 106(3): p. 491-511

10. Gendreau, M. and J.-Y. Potvin, Metaheuristics in combinatorial optimization. Annals of Operations Research, 2005. 140(1): p. 189-213

11. Glover, F., Future paths for integer programming and links to artificial intelligence Computers \& operations research, 1986. 13(5): p. 533-549

12. Glover, F.W. and G.A. Kochenberger, Handbook of metaheuristics. Vol. 57. 2006: Springer Science \& Business Media http://vrp.atd-lab.inf.puc-rio.br/index.php/en/instances?view=vrp13. 
13. Huang, S.-H., Solving the multi-compartment capacitated location routing problem with pickup-delivery routes and stochastic demands. Computers \& Industrial Engineering, 2015. 87: p. 104-113.

14. Jaziri, W., Local search techniques: focus on tabu search. Published by In-The in September, 2008.

Kachitvichyanukul, V., Particle swarm optimization and two solution representations for solving the capacitated vehicle routing problem.Computers \& Industrial Engineering, 2009. 56(1): p. 380-387

15. Kok, A.L., et al., A dynamic programming heuristic for the vehicle routing problem with time windows and European Community social legislation Transportation Science, 2010. 44(4): p. $442-454$

16. Kokash, N., An introduction to heuristic algorithms. Department of Informatics and Telecommunications, 2005: p. 1-8

17. Laporte, G., et al., Classical and modern heuristics for the vehicle routing problem. International transactions in operational research, 2000. 7(4-5):p. 285-300

18. Lin, S.-W., et al., Applying hybrid meta-heuristics for capacitated vehicle routing problem. Expert Systems with Applications, 2009. 36(2):p. 1505-1512.

19. Naddef, D. and G. Rinaldi, Branch-and-cut algorithms for the capacitated VRP, in The vehicle routing problem. 2002, SIAM. p. 53-84

20. Osman, I.H., Metastrategy simulated annealing and tabu search algorithms for the vehicle routing problem. Annals of operation research,1993. 41(4): p. 421-451

21. Peterson, B., et al., Flexible milk-runs for stochastic vehicle routing. 2010

22. Ray, S., Revisiting The Evolution and Application of Assignment Problem Industrial Engineering Letters, 2016. 6(10): p. 16-38

23. Ruttanateerawichien, K., W. Kurutach, and T. Pichpibul. A new efficient and effective golden-ball-based technique for the capacitated vehicle routing problem. in Computer and Information Science (ICIS), 2016 IEEE/ACIS 15th International Conference on. 2016. IEEE

24. Toth, P. and D. Vigo, Branch-and-bound algorithms for the capacitated VRP, in The vehicle routing problem. 2002, SIAM. p. 29-51

25. Zhang, Y., et al., Analysis of an Automated Vehicle Routing Problem in Logistics considering Path Interruption. Journal of Advanced Transportation, 2017. 2017. 